\title{
Pion-nucleon scattering with explicit Delta
} resonance

\section{De-Liang Yao*}

Institute of Modern Physics, Chinese Academy of Sciences, Lanzhou 730000, China

E-mail: dlyao@impcas.ac.cn

Pion-nucleon scattering is one of the most fundamental processes in nuclear and hadron physics, which is of particular importance for our understanding of chiral dynamics of quantum chromodynamics. In this talk, I will review recent progresses on the study of pion-nucleon scattering within the framework of covariant baryon chiral perturbation theory with explicit Delta resonances. Chiral results for elastic reactions will be shown and the effect of the inclusion of explicit Delta resonances will be discussed.

The 9th International workshop on Chiral Dynamics

17-21 September 2018

Durham, NC, USA

${ }^{*}$ Speaker. 


\section{Introduction}

Low-energy pion-nucleon $(\pi N)$ scattering offers an important playground for us to understand the chiral dynamics of quantum chromodynamics. Although the study of elastic $\pi N$ scattering in baryon chiral perturbation theory (BChPT) is now very successful and becomes more and more mature, it suffered a winding development in the past thirty years. The reason is owing to the occurence of power counting breaking (PCB) issue, which was first pointed out in Ref. [1], when the $\overline{\mathrm{MS}}-1$ subtraction scheme was employed together with dimensional regularization (DR) to deal with the loop diagrams. To restore the power counting rule, various approaches have been proposed: heavy baryon (HB) formalism [2, 3], infrared regularization (IR) $[4,5]$ and extended-onmass-shell (EOMS) scheme [6, 7, 8].

Comparison of the above-mentioned approaches is shown in Fig. 1. It is demonstrated in Ref. [5] that the loop integral can be separated into two parts: infrared-singular part with $\log M_{\pi}$ and infrared-regular part which is a polynomial of $M_{\pi}$ and external momenta, where $M_{\pi}$ denotes the mass of the pion fields. In Fig. 1, the infrared-singular and -regular parts are marked in red and blue, respectively. The blue squares are PCB terms, belonging to the regular part. The power counting rule is denoted by the green dashed line in the figure. In the HB approach, only the infrared-singular pieces respecting the naive power counting rule are kept. This is achieved by making a further expansion in terms $1 / m_{B}$, with $m_{B}$ the baryon mass, in addition to the normal chiral expansion. Within this framework $\pi N$ scattering was analysed in detail up to order $\mathscr{O}\left(p^{3}\right)[9,10]$ and later up to order $\mathscr{O}\left(p^{4}\right)$ [11]. In the IR method, all the infrared-regular pieces are dropped and in practice it corresponds to changing the integration domain of Feynman parameters, e.g. $\int_{0}^{1} \mathrm{~d} x \rightarrow \int_{0}^{\infty} \mathrm{d} x$ [5]. By making use of the IR scheme, $\pi N$ scattering has been studied up to $\mathscr{O}\left(p^{4}\right)$ order [12] (see also Ref. [13] for $\mathscr{O}\left(p^{3}\right)$ order calculation). Besides, the analyses of the isospin violation and the $\mathrm{SU}(3)$ sector of BChPT have also been considered in Refs. [14] and [15], respectively.

However, the analytic structure of the obtained chiral amplitude is distorted both in HB and IR schemes, due to the discard of an infinity series of infrared-singular and/or -regular terms [13]. This issue is overcome in EOMS scheme. In EOMS scheme, the PCB terms are absorbed by shifting the low-energy constants (LECs) in the chiral effective Lagrangian, since they are polynomials and have the same analytic structure as the tree amplitudes. $\pi N$ scattering has been calculated using EOMS scheme in Ref. [16] up to order $\mathscr{O}\left(p^{3}\right)$ and in Ref. [17] up to order $\mathscr{O}\left(p^{4}\right)$. Extension beyond

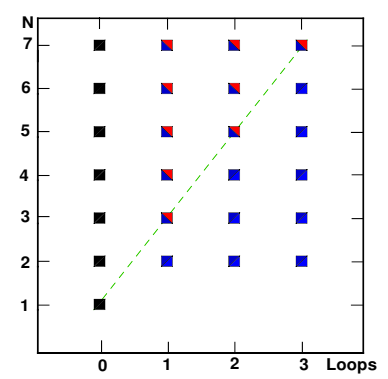

DR ( $\overline{\mathbf{M S}}-1)$

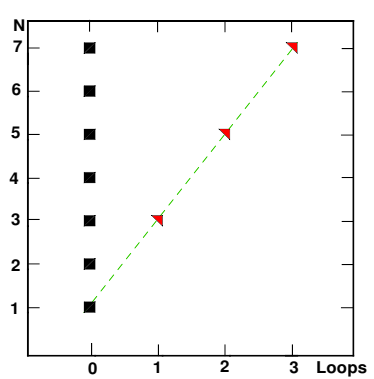

HB

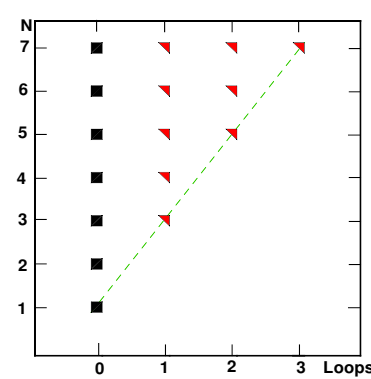

IR

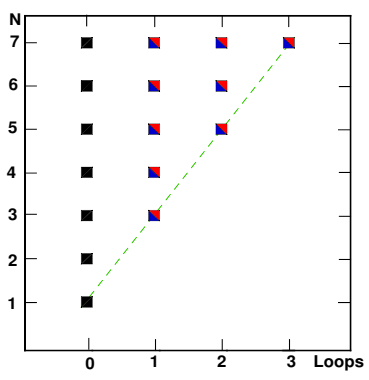

EOMS

Figure 1: Comparison of renormalization schemes of BChPT. The $y$-axis is chiral order $N$ and $x$-axis is the number of loops for a given Feynman diagram. 
the low-energy region is done in Ref. [18]. Contributions to the scattering amplitudes obtained in those works possess the correct power counting and correct analytic properties. Nevertheless, in Refs. $[16,17]$, the contribution of the explicit $\Delta$ resonances is only partially incorporated. The neglected $\Delta$ contributions could be siganificant as indicated by positivity bounds on the LECs [19]. In my recent work [20] to be reviewed in next section, by using EOMS scheme, a complete calculation of elastic $\pi N$ scattering has been carried out for the first time in manifestly relativistic BChPT with explicit $\Delta$ resonances up to leading one-loop order, i.e., next-to-next-to-leading order (NNLO) or $\mathscr{O}\left(p^{3}\right)$.

\section{Elastic $\pi N$ scattering in BChPT with explicit $\Delta$ resonances up to NNLO}

\subsection{Basics for elastic $\pi N$ scattering}

The amplitude for the process of elastic $\pi N$ scattering, denoted by $\pi^{a}(q)+N(p) \rightarrow \pi^{a^{\prime}}\left(q^{\prime}\right)+$ $N\left(p^{\prime}\right)$ for clarity, can be conventionally written as

$$
\begin{aligned}
T_{\pi N}^{a^{\prime} a}(s, t) & =\chi_{N^{\prime}}^{\dagger}\left\{\delta_{a^{\prime} a} T^{+}(s, t)+\frac{1}{2}\left[\tau_{a^{\prime}}, \tau_{a}\right] T^{-}(s, t)\right\} \chi_{N}, \\
T^{ \pm}(s, t) & =\bar{u}^{\left(s^{\prime}\right)}\left(p^{\prime}\right)\left\{D^{ \pm}(s, t)-\frac{1}{4 m_{N}}\left[q^{\prime}, q\right] B^{ \pm}(s, t)\right\} u^{(s)}(p),
\end{aligned}
$$

where $a^{\prime}$ and $a$ are Cartesian isospin indices, $\tau_{i}$ are the Pauli matrices and $\chi_{N}, \chi_{N^{\prime}}$ denote nucleon iso-spinors. Furthermore, the superscript $\left(s^{\prime}\right),(s)$ denote the spins of the Dirac spinors $\bar{u}, u$, respectively. $s, t$ are Mandelstam variables defined by

$$
s=(p+q)^{2}, \quad t=\left(p-p^{\prime}\right)^{2} .
$$

The $D^{ \pm}$and $B^{ \pm}$are unknown functions, which usually can be determined by using dispersion relations under guidance of $S$-matrix arguments or by invoking baryon $\chi \mathrm{PT}$, up to some unknown parameters, i.e., subtraction constants for the former and LECs for the latter. In practice, functions $A^{ \pm} \equiv D^{ \pm}-v B^{ \pm}$, where $v \equiv(s-u) /\left(4 m_{N}\right)$ with $m_{N}$ the physical nucleon mass, are introduced for convenience. Amplitudes with definite isospin can be obtained through

$$
\mathscr{A}^{I=\frac{1}{2}}=\mathscr{A}^{+}+2 \mathscr{A}^{-}, \quad \mathscr{A}^{I=\frac{3}{2}}=\mathscr{A}^{+}-\mathscr{A}^{-},
$$

where $\mathscr{A} \in\{A, B, D\}$ and $I$ denotes isospin quantum number.

The relevant partial wave amplitudes $f_{\ell \pm}^{I}(s)$ are written as

$$
f_{\ell \pm}^{I}(s)=\frac{1}{16 \pi \sqrt{s}}\left\{E_{p}^{+}\left[A_{\ell}^{I}(s)+\left(\sqrt{s}-m_{N}\right) B_{\ell}^{I}(s)\right]+E_{p}^{-}\left[-A_{\ell \pm 1}^{I}(s)+\left(\sqrt{s}+m_{N}\right) B_{\ell \pm 1}^{I}(s)\right]\right\}
$$

with $E_{p}^{ \pm}=\frac{s+m_{N}^{2}-M_{\pi}^{2}}{2 \sqrt{s}} \pm m_{N}$ and the subscript $\ell \pm$ is an abbreviation for the total angular momentun $J=\ell \pm \frac{1}{2}$. One popular notation for all the partial waves is the spectroscopic one, $L_{2 I, 2 J}$, with $L=S, P, D, F, \ldots$ (corresponding to $\ell=0,1,2,3, \ldots$ ). The partial wave projection of the isospin amplitudes is given by

$$
\mathscr{A}_{\ell}^{I}(s)=\int_{-1}^{+1} \mathscr{A}^{I}\left(s, t\left(s, z_{s}\right)\right) P_{\ell}\left(z_{s}\right) \mathrm{d} z_{s}, \quad z_{s} \equiv \cos \theta,
$$

where $\theta$ is the scatting angle in the center-of-mass (CMS) frame and $P_{\ell}\left(z_{s}\right)$ are Legendre polynomials. 


\subsection{Chiral effective Lagrangians}

For the calculation of the $\pi N$ scattering amplitude in BChPT up to order $\mathscr{O}\left(p^{3}\right)$ the following chiral effective Lagrangians are needed

$$
\mathscr{L}_{\mathrm{eff}}=\sum_{i=1}^{2} \mathscr{L}_{\pi \pi}^{(2 i)}+\sum_{j=1}^{3} \mathscr{L}_{\pi N}^{(j)}+\sum_{k=1}^{2} \mathscr{L}_{\pi \Delta}^{(k)}+\sum_{l=1}^{3} \mathscr{L}_{\pi N \Delta}^{(l)},
$$

where the relevant pieces at different chiral orders are given by [21, 22, 10, 23]

$$
\begin{aligned}
& \mathscr{L}_{\pi \pi}^{(2)}=\frac{F^{2}}{4} \operatorname{Tr}\left(\partial_{\mu} U \partial^{\mu} U^{\dagger}\right)+\frac{F^{2} M^{2}}{4} \operatorname{Tr}\left(U^{\dagger}+U\right), \\
& \mathscr{L}_{\pi \pi}^{(4)}=\frac{1}{8} l_{4}\left\langle u^{\mu} u_{\mu}\right\rangle\left\langle\chi_{+}\right\rangle+\frac{1}{16}\left(l_{3}+l_{4}\right)\left\langle\chi_{+}\right\rangle^{2}, \\
& \mathscr{L}_{\pi N}^{(1)}=\bar{\Psi}_{N}\left\{i \not D-m+\frac{1}{2} g \not \gamma^{5}\right\} \Psi_{N} \\
& \mathscr{L}_{\pi N}^{(2)}=\bar{\Psi}_{N}\left\{c_{1}\left\langle\chi_{+}\right\rangle-\frac{c_{2}}{4 m^{2}}\left\langle u^{\mu} u^{v}\right\rangle\left(D_{\mu} D_{v}+\text { h.c. }\right)+\frac{c_{3}}{2}\left\langle u^{\mu} u_{\mu}\right\rangle-\frac{c_{4}}{4} \gamma^{\mu} \gamma^{v}\left[u_{\mu}, u_{v}\right]\right\} \Psi_{N} \\
& \mathscr{L}_{\pi N}^{(3)}=\bar{\Psi}_{N}\left\{-\frac{d_{1}+d_{2}}{4 m}\left(\left[u_{\mu},\left[D_{v}, u^{\mu}\right]+\left[D^{\mu}, u_{v}\right]\right] D^{v}+\text { h.c. }\right)\right. \\
& +\frac{d_{3}}{12 m^{3}}\left(\left[u_{\mu},\left[D_{v}, u_{\lambda}\right]\right]\left(D^{\mu} D^{v} D^{\lambda}+\text { sym. }\right)+\text { h.c. }\right)+i \frac{d_{5}}{2 m}\left(\left[\chi_{-}, u_{\mu}\right] D^{\mu}+\text { h.c. }\right) \\
& +i \frac{d_{14}-d_{15}}{8 m}\left(\sigma^{\mu v}\left\langle\left[D_{\lambda}, u_{\mu}\right] u_{v}-u_{\mu}\left[D_{v}, u_{\lambda}\right]\right\rangle D^{\lambda}+h . c .\right)+\frac{d_{16}}{2} \gamma^{\mu} \gamma^{5}\left\langle\chi_{+}\right\rangle u_{\mu} \\
& \left.+\frac{i d_{18}}{2} \gamma^{\mu} \gamma^{5}\left[D_{\mu}, \chi_{-}\right]\right\} \Psi_{N} \\
& \mathscr{L}_{\pi \Delta}^{(1)}=-\bar{\Psi}_{\mu}^{i} \xi_{i j}^{\frac{3}{2}}\left\{\left(i \not D^{j k}-m_{\Delta} \delta^{j k}\right) g^{\mu v}+i A\left(\gamma^{\mu} D^{v, j k}+\gamma^{v} D^{\mu, j k}\right)\right. \\
& +\frac{i}{2}\left(3 A^{2}+2 A+1\right) \gamma^{\mu} \not D^{j k} \gamma^{v}+m_{\Delta} \delta^{j k}\left(3 A^{2}+3 A+1\right) \gamma^{\mu} \gamma^{v} \\
& \left.+\frac{g_{1}}{2} \psi^{j k} \gamma_{5} g^{\mu v}+\frac{g_{2}}{2}\left(\gamma^{\mu} u^{v, j k}+u^{v, j k} \gamma^{\mu}\right) \gamma_{5}+\frac{g_{3}}{2} \gamma^{\mu} \mu^{j k} \gamma_{5} \gamma^{v}\right\} \xi_{k l}^{\frac{3}{2}} \Psi_{v}^{l}, \\
& \mathscr{L}_{\pi \Delta}^{(2)}=a_{1} \bar{\Psi}_{\mu}^{i} \xi_{i j}^{\frac{3}{2}} \Theta^{\mu \alpha}(z)\left\langle\chi_{+}\right\rangle \delta^{j k} g_{\alpha \beta} \Theta^{\beta v}\left(z^{\prime}\right) \xi_{k l}^{\frac{3}{2}} \Psi_{v}^{l}, \\
& \mathscr{L}_{\pi N \Delta}^{(1)}=h \bar{\Psi}_{\mu}^{i} \xi_{i j}^{\frac{3}{2}} \Theta^{\mu \alpha}\left(z_{1}\right) \omega_{\alpha}^{j} \Psi_{N}+\text { h.c. }, \\
& \mathscr{L}_{\pi N \Delta}^{(2)}=\bar{\Psi}_{\mu}^{i} \xi_{i j}^{\frac{3}{2}} \Theta^{\mu \alpha}\left(z_{2}\right)\left[i b_{3} \omega_{\alpha \beta}^{j} \gamma^{\beta}+i \frac{b_{8}}{m} \omega_{\alpha \beta}^{j} i D^{\beta}\right] \Psi_{N}+\text { h.c. }, \\
& \mathscr{L}_{\pi N \Delta}^{(3)}=\bar{\Psi}_{\mu}^{i} \xi_{i j}^{\frac{3}{2}} \Theta^{\mu v}\left(z_{3}\right)\left[\frac{f_{1}}{m}\left[D_{v}, \omega_{\alpha \beta}^{j}\right] \gamma^{\alpha} i D^{\beta}-\frac{f_{2}}{2 m^{2}}\left[D_{v}, \omega_{\alpha \beta}^{j}\right]\left\{D^{\alpha}, D^{\beta}\right\}\right. \\
& \left.+f_{4} \omega_{v}^{j}\left\langle\chi_{+}\right\rangle+f_{5}\left[D_{v}, i \chi_{-}^{j}\right]\right] \Psi_{N}+\text { h.c.. }
\end{aligned}
$$

In the above Lagrangians, pion fields are contained in the $U$ matrix, the nucleon and $\Delta$ fields are indicated by $\Psi_{N}$ and $\Psi_{\mu}^{i}$ respectively. Here \langle\rangle denotes the trace in flavor space, $F$ is the pion decay constant in the chiral limit, and $l_{3}, l_{4}$ are mesonic LECs. Furthermore, $m$ and $g$ denote the nucleon bare mass and the bare axial coupling constant, respectively. The LECs $c_{i}$ and $d_{j}$ have dimension $\mathrm{GeV}^{-1}$ and $\mathrm{GeV}^{-2}$, respectively. $m_{\Delta}$ and $g_{1}, g_{2}, g_{3}, a_{1}$ are the bare mass of the delta 
and bare coupling constants, respectively. Further, $\Theta^{\mu \alpha}=g^{\mu \alpha}+z \gamma^{\mu} \gamma^{\nu}$, where $z$ is a so-called offshell parameter. Finally, the bare pion-nucleon-delta coupling constant at lowest order is denoted by $h$ and $b_{3}, b_{8}, f_{1}, f_{2}, f_{4}$ and $f_{5}$ are bare LECs of higher orders. Note that in fact the operators concerning $b_{3}, b_{8}, f_{1}, f_{2}, f_{4}$ and $f_{5}$ are redundant in the sense that their contributions can be taken into account by the redefinition of $h$ in the leading-order delta exchanges and the LECs in the contact terms [20]. The parameters $z_{1}, z_{2}$ and $z_{3}$ are off-shell parameters in the $\pi N \Delta$ interactions. However, they do not contribute in physical quantities. Definitions on covariant derivatives and chiral operators are referred to Ref. [20].

\subsection{Calculation procedure}

The procedure of calculating the $\pi N$ scattering amplitudes, or the $A, B$ functions, is explained in the following. First, all the relevant Feynman rules are derived from the chiral effective Lagrangians given in the previous subsection. Here I list all of them in the appendix for easy reference. Second, all the possible one-particle irreducible topologies, i.e., Feynman diagrams, are drawn (see Fig. 1-3 in Ref. [20]), with the help of the so-called small-scale-expansion (SSE) power counting rule [24, 23]. In SSE, the chiral order of the $\Delta$ propagator is counted the same as the one of the nucleon propagator. Third, the scattering amplitudes are calculated diagram by diagram by inserting the Feynman rules into the topologies. Usually, this can be easily done by computer using the popular Mathematica package FeynCalc [25, 26]. However, here I use a more efficient Mathematica package called AmpCalc written by myself and Feng-Kun Guo [27]. Fourth, wave function renormalzation is performed to get the full one-loop scattering amplitudes. The one-loop amplitudes are expressed in terms scalar one-loop integrals and then simultaneously expanded in terms of small parameters, $M_{\pi}, s-m_{N}^{2}$ and $t$. The ultraviolet (UV) divergences and the PCB terms are cancelled order by order by shifting the LECs in the tree amplitudes, which leads to

$$
\begin{aligned}
& X \equiv X_{R}+\frac{\bar{\delta} X}{16 \pi^{2} F^{2}} R+\frac{\overline{\bar{\delta}} X}{16 \pi^{2} F^{2}}, \quad X \in\left\{g, h, m, m_{\Delta}, a_{1}, c_{i=1,4}\right\} \\
& Y \equiv Y_{R}+\frac{\bar{\delta} Y}{16 \pi^{2} F^{2}} R, \quad Y \in\left\{\ell_{3}, \ell_{4}, d_{1}+d_{2}, d_{3}, d_{5}, d_{14}-d_{15}, d_{18}-2 d_{16}\right\}
\end{aligned}
$$

where $X_{R}$ and $Y_{R}$ are renormalized parameters and $R \equiv 2 /(d-4)+\gamma_{E}-1-\ln (4 \pi)$, and $\gamma_{E}$ is the Euler number. The subscript $R$ is always omitted if there is no confusion caused.

\subsection{Results}

The $S$ - and $P$-wave phase shifts generated by the recent Roy-Steiner-equation analysis (RS) of the $\pi N$ scattering [29], with both the central values and the errors of results given by Schenk-like or conformal parameterizations, are fitted with the delta-less (i.e., Fit-I: up to $1.11 \mathrm{GeV}$ ) and delta-full (i.e., Fit-III: up to $1.2 \mathrm{GeV}$ for $P_{33}$ and up to $1.11 \mathrm{GeV}$ for the other waves) BChPT results. The fitting results are displayed in Fig. 2. Fit-III improves the predictions beyond fitting ranges in most of the partial waves due to the inclusion of the delta contribution. Predictions on the scattering lengths and higher-order partial-wave phase shifts are also obtained, for more details see Ref. [20]. 

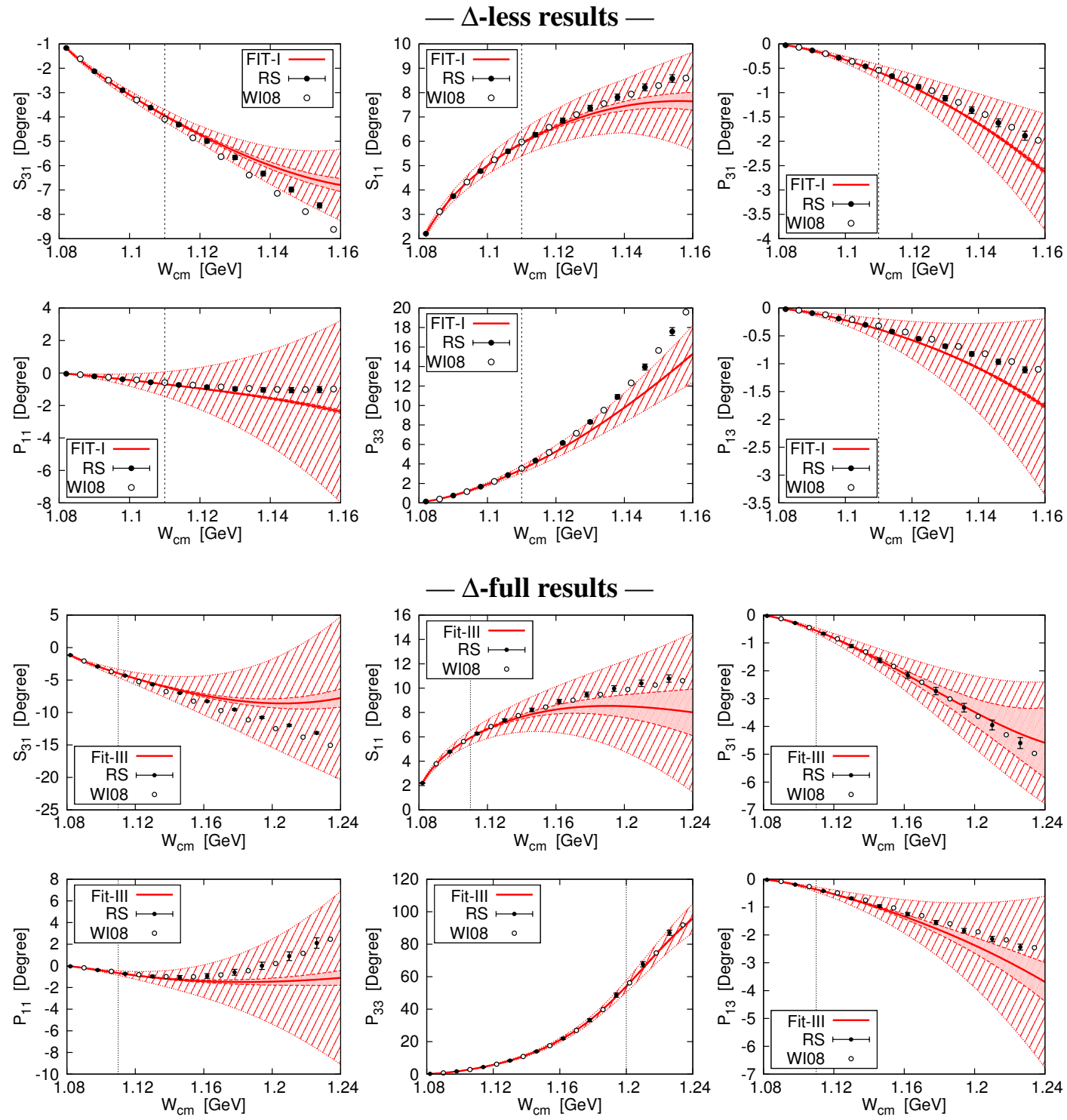

Figure 2: Phase shifts obtained from delta-less and delta-full BChPT. Dots with error bars correspond to the RS phase shifts, while circles without error bars stand for the GWU phase shifts. The solid (red) lines represent the results of the current work. The red narrow error bands correspond to the uncertainties propagated from the errors of LECs. The wide dashed error bands correspond to the theoretical uncertainties due to the truncation of the chiral series estimated by using the approach proposed in Ref. [28].

\section{Further studies and applications}

In the same framework as discussed in the previous section, the study of elastic $\pi N$ scattering has been extended up to one-order higher, i.e. next-to-next-to-next-to-leading order (NNNLO), by performing a combined analysis of $\pi N \rightarrow \pi N$ and $\pi N \rightarrow \pi \pi N$ reactions in Ref. [30]. For easy comparison to HB results, a modified EOMS scheme has been employed, which only affects the values of the LECs at the highest order under consideration. In addition, experimental data on 
differential cross sections and polarizations, instead of phase shifts, are fitted in order to determine the LECs more reliably, though only tree-level contribution of $\Delta$ resonances is included.

On the other hand, the values of the LECs obtained in Ref. [20], especially the ones relevant to $\Delta$ interactions, are applied to the extraction of nucleon axial charge and radius [31], to the calculations of the cross sections of the weak pion productions [32, 33], etc..

\section{Summary and outlook}

I have reviewed the study of $\pi N$ scattering in a covariant BChPT with $\Delta$ resonances as explicit degrees of freedom. Experimental data and phase-shift data can be well described by the obtained chiral results. The determined values of the LECs are important for the predictions of other relevant physical quantities or processes in the same framework. Future investigation on the explicit inclusion of Roper resonance in $\pi N$ scattering should be important for the inelastic case.

\section{Acknowledgements}

I thank all my collaborators for their dedicating to the project discussed here.

\section{Appendix: Feynman rules}

Based on the effective Lagrangians, it is straightforward to derive the Feynman rules that are relevant to the calculation of $\pi N$ scattering up to and including $\mathscr{O}\left(p^{3}\right)$. In this appendix, all of them are listed item by item, for the sake of easy references.

\section{A.1 Propagators}

- Pion propagator: an incoming pion with momentum $q$ and isospin index $a$, an outgoing pion with momentum $q$ and isospin index $b$

$$
i \Delta_{F}^{a b}(q)=\frac{i \delta^{a b}}{q^{2}-M^{2}+i 0^{+}} .
$$

- Nucleon propagator: an incoming nucleon with momentum $p$, an outgoing nucleon with momentum $p$

$$
i S_{F}(p)=\frac{i}{\not p-m+i 0^{+}}
$$

- Delta propagator ( $d$ dimension and $A=-1$ ): an outgoing delta with momentum $p$, Dirac index $\mu$ and isospin index $i$, an incoming delta with momentum $p$, Dirac index $v$, and isospin index $j$,

$$
\begin{gathered}
i S_{F, i j}^{\mu v}(p)=-\frac{i\left(\not p+m_{\Delta}\right)}{p^{2}-m_{\Delta}^{2}+i 0^{+}}\left[g^{\mu v}-\frac{1}{d-1} \gamma^{\mu} \gamma^{v}+\frac{1}{(d-1) m_{\Delta}}\left(p^{\mu} \gamma^{v}-\gamma^{\mu} p^{v}\right)\right. \\
\left.-\frac{d-2}{(d-1) m_{\Delta}^{2}} p^{\mu} p^{v}\right] \xi_{i j}^{\frac{3}{2}}
\end{gathered}
$$




\section{A.2 Pion self-interacting vertex}

- $\mathscr{O}\left(p^{2}\right), \pi \pi$ : two incoming pions (momenta $q_{a}$ and $q_{b}$, isospins $a$ and $b$, in order)

$$
-\frac{2 i M^{2}}{F^{2}}\left[\left(\ell_{3}+\ell_{4}\right) M^{2}+\ell_{4} q_{a} \cdot q_{b}\right] \delta_{a b} .
$$

- $\mathscr{O}\left(p^{2}\right), \pi \pi \pi \pi$ : four incoming pions (momenta $q_{a}, q_{b}, q_{c}$ and $q_{d}$, isospins $a, b, c$ and $d$, in order)

$$
\begin{aligned}
& \frac{i}{3 F^{2}}\left\{\quad \delta_{a b} \delta_{c d}\left[2\left(q_{a} \cdot q_{b}+q_{c} \cdot q_{d}\right)-\left(q_{a}+q_{b}\right) \cdot\left(q_{c}+q_{d}\right)+M^{2}\right]\right. \\
& +\delta_{a c} \delta_{b d}\left[2\left(q_{a} \cdot q_{c}+q_{b} \cdot q_{d}\right)-\left(q_{a}+q_{c}\right) \cdot\left(q_{b}+q_{d}\right)+M^{2}\right] \\
& \left.+\delta_{a d} \delta_{b c}\left[2\left(q_{a} \cdot q_{d}+q_{b} \cdot q_{c}\right)-\left(q_{a}+q_{d}\right) \cdot\left(q_{b}+q_{c}\right)+M^{2}\right]\right\} \text {. }
\end{aligned}
$$

\section{A.3 Pion-nucleon interacting vertices}

- $\mathscr{O}\left(p^{2}\right), N N$ : an outgoing nucleon and an incoming nucleon,

$$
4 i c_{1} M^{2} \text {. }
$$

- $\pi N N$ : an outgoing nucleon, an incoming nucleon and an incoming pion (momentum $q_{a}$, isospin $a$ ),

$$
\begin{aligned}
\mathscr{O}(\varepsilon): & -\frac{g}{2 F} \phi_{a} \gamma^{5} \tau_{a}, \\
\mathscr{O}\left(\varepsilon^{3}\right): & \frac{d_{18}-2 d_{16}}{F} M^{2} \phi_{a} \gamma^{5} \tau_{a} .
\end{aligned}
$$

- $\pi \pi N N$ : an outgoing nucleon (momentum $p^{\prime}$ ), an incoming nucleon (momentum $p$ ) and two incoming pion (momenta $q_{a}$ and $q_{b}$, isospins $a$ and $b$, in order),

$$
\begin{aligned}
& \mathscr{O}(\varepsilon): \quad \frac{1}{4 F^{2}} \varepsilon_{a b c} \tau_{c}\left(\phi_{a}-q_{b}\right), \\
& \mathscr{O}\left(\varepsilon^{2}\right): \quad-\frac{1}{F^{2}}\left\{4 i c_{1} M^{2}+\frac{c_{2}}{m^{2}}\left(p^{\prime} \cdot q_{a} p^{\prime} \cdot q_{b}+p \cdot q_{a} p \cdot q_{b}\right)+2 i c_{3} q_{a} \cdot q_{b}\right\} \delta_{a b} \\
& +\frac{i c_{4}}{F^{2}} \varepsilon_{a b c} \tau_{c} \sigma_{\mu v} q_{a}^{\mu} q_{b}^{v} \\
& \mathscr{O}\left(\varepsilon^{3}\right): \quad \frac{1}{m F^{2}}\left\{\left[\left(d_{1}+d_{2}\right) q_{a} \cdot q_{b}\left(q_{b}-q_{a}\right) \cdot p^{\prime}+\frac{d_{3}}{m^{2}} p^{\prime} \cdot q_{a} p^{\prime} \cdot q_{b} p^{\prime} \cdot\left(q_{b}-q_{a}\right)\right.\right. \\
& \left.-2 d_{5} M^{2} p^{\prime} \cdot\left(q_{b}-q_{a}\right)\right] \varepsilon_{a b c} \tau_{c} \\
& \left.-\frac{d_{14}-d_{15}}{2} \sigma_{\mu v}\left(q_{a}^{\lambda} q_{a}^{\mu} q_{b}^{v}+q_{a}^{v} q_{b}^{\lambda} q_{b}^{\mu}\right) p_{\lambda}^{\prime} \delta_{a b}\right\}+\left(p^{\prime} \rightarrow p\right) \text {. }
\end{aligned}
$$

- $\mathscr{O}(p), 3 \pi N N$ : an incoming nucleon, an outgoing nucleon and three incoming pions (momenta $q_{a}, q_{b}$ and $q_{c}$, isospins $a, b$ and $c$, in order),

$$
\frac{g}{12 F^{3}}\left[\tau_{a} \delta_{b c}\left(2 q_{a}-q_{b}-q_{c}\right)+\tau_{b} \delta_{a c}\left(2 q_{b}-q_{a}-q_{c}\right)+\tau_{c} \delta_{a b}\left(2 q_{c}-q_{a}-q_{b}\right)\right] \gamma^{5}
$$


- $\mathscr{O}(p), 4 \pi N N$ : an incoming nucleon, an outgoing nucleon and four incoming pions (momenta $q_{a}, q_{b} q_{c}$ and $q_{d}$, isospins $a, b, c$ and $d$, in order),

$$
\begin{aligned}
-\frac{1}{24 F^{4}} & {\left[\delta_{a b} \varepsilon_{c d e} \tau_{e}\left(q_{c}-q_{d}\right)+\delta_{a c} \varepsilon_{b d e} \tau_{e}\left(q_{b}-q_{d}\right)+\delta_{a d} \varepsilon_{b c e} \tau_{e}\left(q_{b}-q_{c}\right)\right.} \\
& \left.+\delta_{b c} \varepsilon_{a d e} \tau_{e}\left(q_{a}-q_{d}\right)+\delta_{b d} \varepsilon_{a c e} \tau_{e}\left(\phi_{a}-q_{c}\right)+\delta_{c d} \varepsilon_{a b e} \tau_{e}\left(q_{a}-q_{b}\right)\right] .
\end{aligned}
$$

\section{A.4 Pion-delta interacting vertices (here set $A=-1$, hence $g_{1}=-g_{2}=-g_{3}$ )}

- $\mathscr{O}\left(p^{2}\right), \Delta \Delta$ : an outgoing delta ( $\operatorname{spin} \mu$, isospin $\left.i\right)$, an incoming delta (spin $v$, isospin $j$ )

$$
4 i M^{2} \Theta_{\mu \mu^{\prime}}(z) \Theta^{\mu^{\prime} v}(z) \xi_{i j}^{\frac{3}{2}} .
$$

- $\mathscr{O}(p), \pi \Delta \Delta$ : an outgoing delta ( $\operatorname{spin} \mu$, isospin $i)$, an incoming delta (spin $v$, isospin $n$ ) and an incoming pion (momentum $q_{a}$, isospin $a$ ),

$$
\frac{g_{1}}{2 F^{2}} \xi_{i j}^{\frac{3}{2}} \tau_{a} \xi_{j n}^{\frac{3}{2}}\left\{q_{a} \gamma_{5} g^{\mu v}-\left(\gamma^{\mu} q_{a}^{v}+q_{a}^{\mu} \gamma^{v}\right) \gamma_{5}-\gamma^{\mu} \phi_{a} \gamma_{5} \gamma^{v}\right\}
$$

- $\mathscr{O}(p), \pi \pi \Delta \Delta$ : an outgoing delta (Dirac $\mu$, isospin $i)$, an incoming delta (Dirac $v$, isospin $n$ ) and two incoming pions (momenta $q_{a}$ and $q_{b}$, isospins $a$ and $b$, in order),

$$
\begin{aligned}
& \left\{\frac{i}{2 F^{2}}\left(\xi_{i a}^{\frac{3}{2}} \xi_{b n}^{\frac{3}{2}}-\xi_{i b}^{\frac{3}{2}} \xi_{a n}^{\frac{3}{2}}\right)-\frac{1}{4 F^{2}} \xi_{i j}^{\frac{3}{2}} \varepsilon_{a b c} \tau_{c} \xi_{j n}^{\frac{3}{2}}\right\} \times \\
& \left\{\left(\phi_{a}-q_{b}\right) g^{\mu v}-\left[\gamma^{\mu}\left(q_{a}-q_{b}\right)^{v}+\gamma^{v}\left(q_{a}-q_{b}\right)^{\mu}\right]+\gamma^{\mu}\left(q_{a}-q_{b}\right) \gamma^{v}\right\} .
\end{aligned}
$$

\section{A.5 Pion-nucleon-delta interacting vertices}

- $\pi \Delta N$ : an outgoing delta (Dirac $\mu$, isospin $i$ ), an incoming nucleon (momentum $p$ ) and an incoming pion (momentum $q_{a}$, isospin $a$ ),

$$
\begin{aligned}
& \mathscr{O}(p):-\frac{g_{\pi N \Delta}}{F}\left(q_{a}^{\mu}+z_{1} \gamma^{\mu} \phi_{a}\right) \xi_{i a}^{\frac{3}{2}} \\
& \mathscr{O}\left(p^{2}\right):-\frac{1}{F}\left(q_{a}^{\mu}+z_{2} \gamma^{\mu} \phi_{a}\right)\left\{b_{3} \phi_{a}+\frac{b_{8}}{m} p \cdot q_{a}\right\} \xi_{i a}^{\frac{3}{2}} \\
& \mathscr{O}\left(\varepsilon^{3}\right):-\frac{1}{F}\left(q_{a}^{\mu}+z_{3} \gamma^{\mu} \phi_{a}\right)\left\{\frac{1}{m}\left[f_{1} \phi_{a}+\frac{f_{2}}{m} p \cdot q_{a}\right] p \cdot q_{a}-2 M^{2}\left(2 f_{4}-f_{5}\right)\right\} \xi_{i a}^{\frac{3}{2}} .
\end{aligned}
$$

- $\pi N \Delta$ : an outgoing nucleon, an incoming delta (Dirac $\mu$, isospin $i$ ), and an incoming pion (momentum $q_{a}$, isospin $a$ ),

$$
\begin{array}{ll}
\mathscr{O}(p): & -\frac{g_{\pi N \Delta}}{F}\left(q_{a}^{\mu}+z_{1} \phi_{a} \gamma^{\mu}\right) \xi_{a i}^{\frac{3}{2}}, \\
\mathscr{O}\left(p^{2}\right) & \frac{1}{F}\left\{b_{3} \phi_{a}+\frac{b_{8}}{m} p \cdot q_{a}\right\}\left(q_{a}^{\mu}+z_{2} \phi_{a} \gamma^{\mu}\right) \xi_{a i}^{\frac{3}{2}}, \\
\mathscr{O}\left(p^{3}\right) & \frac{1}{F}\left\{\frac{1}{m}\left[f_{1} \phi_{a}+\frac{f_{2}}{m} p \cdot q_{a}\right] p \cdot q_{a}-2 M^{2}\left(2 f_{4}-f_{5}\right)\right\}\left(q_{a}^{\mu}+z_{3} \phi_{a} \gamma^{\mu}\right) \xi_{a i}^{\frac{3}{2}} .
\end{array}
$$


- $\mathscr{O}(p), 3 \pi \Delta N$ : an outgoing delta (spin $\mu$, isospin $i$ ), an incoming nucleon and three incoming pions (momenta $q_{a}, q_{b}$ and $q_{c}$, isospins $a, b$ and $c$, in order),

$$
\begin{aligned}
\frac{g_{\pi N \Delta}}{6 F^{3}}\left(g^{\mu \nu}+z_{1} \gamma^{\mu} \gamma^{v}\right)\{ & \xi_{i a}^{\frac{3}{2}} \delta_{b c}\left(2 q_{a}-q_{b}-q_{c}\right)_{v} \\
& \left.+\xi_{i b}^{\frac{3}{2}} \delta_{a c}\left(2 q_{b}-q_{a}-q_{c}\right)_{v}+\xi_{i c}^{\frac{3}{2}} \delta_{a b}\left(2 q_{c}-q_{a}-q_{b}\right)_{v}\right\}
\end{aligned}
$$

- $\mathscr{O}(p), 3 \pi N \Delta$ : an incoming nucleon, an outgoing delta (spin $\mu$, isospin $i$ ) and three incoming pions (momenta $q_{a}, q_{b}$ and $q_{c}$, isospins $a, b$ and $c$, in order),

$$
\begin{gathered}
\frac{g_{\pi N \Delta}}{6 F^{3}}\left\{\xi_{a i}^{\frac{3}{2}} \delta_{b c}\left(2 q_{a}-q_{b}-q_{c}\right)_{v}+\xi_{b i}^{\frac{3}{2}} \delta_{a c}\left(2 q_{b}-q_{a}-q_{c}\right)_{v}\right. \\
\left.+\xi_{c i}^{\frac{3}{2}} \delta_{a b}\left(2 q_{c}-q_{a}-q_{b}\right)_{v}\right\}\left(g^{v \mu}+z_{1} \gamma^{v} \gamma^{\mu}\right) .
\end{gathered}
$$

In the end, it is worthwhile to note that, the isospin- $\frac{3}{2}$ projector $\xi_{i j}^{\frac{3}{2}}$ occurring in the vertices, which involves the $\Delta(1232)$ resonances, can be substituted by the isospin object $\delta_{i j}$. Taking the $\pi \Delta N$ interacting vertices for example, it can also be rewritten as

$$
-\frac{g_{\pi N \Delta}}{F}\left(q_{a}^{\mu}+z_{1} \gamma^{\mu} \phi_{a}\right) \delta_{i a} .
$$

This is due to the fact that the $\Delta(1232)$-involving vertices in our consideration must be connected by delta propagators and the equivalence is guaranteed by the property of projection operator, i.e. $\xi_{i j}^{\frac{3}{2}} \xi_{j k}^{\frac{3}{2}}=\xi_{i k}^{\frac{3}{2}}=\delta_{i j} \xi_{j k}^{\frac{3}{2}}$.

\section{References}

[1] J. Gasser, M. E. Sainio and A. Svarc, Nucl. Phys. B 307, 779 (1988).

[2] E. E. Jenkins and A. V. Manohar, Phys. Lett. B 255, 558 (1991).

[3] V. Bernard, N. Kaiser, J. Kambor and U. G. Meißner, Nucl. Phys. B 388, 315 (1992).

[4] P. J. Ellis and H. B. Tang, Phys. Rev. C 57, 3356 (1998).

[5] T. Becher and H. Leutwyler, Eur. Phys. J. C 9, 643 (1999).

[6] J. Gegelia and G. Japaridze, Phys. Rev. D 60, 114038 (1999).

[7] J. Gegelia, G. Japaridze and X. Q. Wang, J. Phys. G 29, 2303 (2003).

[8] T. Fuchs, J. Gegelia, G. Japaridze and S. Scherer, Phys. Rev. D 68, 056005 (2003).

[9] M. Mojzis, Eur. Phys. J. C 2, 181 (1998).

[10] N. Fettes, U. G. Meißner and S. Steininger, Nucl. Phys. A 640, 199 (1998).

[11] N. Fettes and U. G. Meißner, Nucl. Phys. A 676, 311 (2000).

[12] T. Becher and H. Leutwyler, JHEP 0106, 017 (2001).

[13] J. M. Alarcon, J. Martin Camalich, J. A. Oller and L. Alvarez-Ruso, Phys. Rev. C 83, 055205 (2011) Erratum: [Phys. Rev. C 87, no. 5, 059901 (2013)]. 
[14] M. Hoferichter, B. Kubis and U. G. Meißner, Nucl. Phys. A 833, 18 (2010).

[15] M. Mai, P. C. Bruns, B. Kubis and U. G. Meißner, Phys. Rev. D 80, 094006 (2009).

[16] J. M. Alarcon, J. Martin Camalich and J. A. Oller, Annals Phys. 336, 413 (2013).

[17] Y. H. Chen, D. L. Yao and H. Q. Zheng, Phys. Rev. D 87, 054019 (2013).

[18] E. Epelbaum, J. Gegelia, U. G. Meißner and D. L. Yao, Eur. Phys. J. C 75, no. 10, 499 (2015).

[19] J. J. Sanz-Cillero, D. L. Yao and H. Q. Zheng, Eur. Phys. J. C 74, 2763 (2014).

[20] D. L. Yao, D. Siemens, V. Bernard, E. Epelbaum, A. M. Gasparyan, J. Gegelia, H. Krebs and U. G. Meißner, JHEP 1605, 038 (2016).

[21] J. Gasser and H. Leutwyler, Annals Phys. 158, 142 (1984).

[22] S. Bellucci, J. Gasser and M. E. Sainio, Nucl. Phys. B 423, 80 (1994) Erratum: [Nucl. Phys. B 431, 413 (1994)].

[23] T. R. Hemmert, B. R. Holstein and J. Kambor, J. Phys. G 24, 1831 (1998).

[24] T. R. Hemmert, B. R. Holstein and J. Kambor, Phys. Lett. B 395, 89 (1997).

[25] R. Mertig, M. Bohm and A. Denner, Comput. Phys. Commun. 64, 345 (1991).

[26] V. Shtabovenko, R. Mertig and F. Orellana, Comput. Phys. Commun. 207, 432 (2016).

[27] $* * *$ Non-standard form, no INSPIRE lookup performed $* * *$

[28] E. Epelbaum, H. Krebs and U. G. Meißner, Eur. Phys. J. A 51, no. 5, 53 (2015).

[29] M. Hoferichter, J. Ruiz de Elvira, B. Kubis and U. G. Meißner, Phys. Rept. 625, 1 (2016) [arXiv:1510.06039 [hep-ph]].

[30] D. Siemens, V. Bernard, E. Epelbaum, A. M. Gasparyan, H. Krebs and U. G. Meißner, Phys. Rev. C 96, no. 5, 055205 (2017) [arXiv:1704.08988 [nucl-th]].

[31] D. L. Yao, L. Alvarez-Ruso and M. J. Vicente-Vacas, Phys. Rev. D 96, no. 11, 116022 (2017).

[32] D. L. Yao, L. Alvarez-Ruso, A. N. Hiller Blin and M. J. Vicente Vacas, Phys. Rev. D 98, no. 7 , 076004 (2018).

[33] D. L. Yao, L. Alvarez-Ruso and M. J. Vicente Vacas, arXiv:1901.00773 [hep-ph]. 